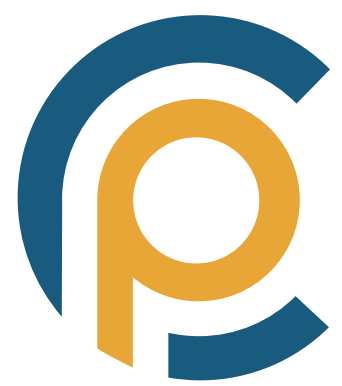

\title{
Aplicaciones Metodológicas Inclusivas para la Enseñanza del Inglés como Lengua Extranjera en Alumnos con Dislexia
}

\author{
Inclusive Methodological Implementations for Teaching \\ English as a Foreign Language to Students with Dyslexia
}

Recibido: 17/03/2020 | Revisado: 20/04/2020 | Aceptado: 02/06/2020 | Publicado: 30/06/2020

\author{
Silvia Fombella Pedrero \\ Universidad Internacional de La Rioja \\ silvia.fombella@gmail.com \\ https://orcid.org/0000-0003-0601-2523
}

\section{Patricia Solís García}

Universidad Internacional de La Rioja

patricia.solis@unir.net

https://orcid.org/0000-0002-2962-5819

\begin{abstract}
Resumen: La dislexia es un trastorno incluido dentro de los Trastornos del Aprendizaje con gran repercusión en todas las áreas curriculares, especialmente en aquellas en las que la competencia comunicativa es altamente desarrollada, como es el caso de la primera lengua extranjera, el inglés. Este idioma está cada vez más extendido en nuestras aulas con la evolución de los centros bilingües. A pesar de adaptar la metodología general que se emplea en la asignatura de inglés, cuando nos encontramos con alumnos con dislexia no se realizan adaptaciones específicas o bien se aplican las medidas existentes expedidas por la inspección educativa. Con la presente investigación se pretende recopilar las medidas metodológicas aportadas por la inspección educativa y por algunos profesionales en ejercicio con el fin último de proporcionar la mejor educación posible a estos alumnos de manera inclusiva. Se ha procedido a analizar los casos de tres alumnos diferentes, entrevistando tanto a padres como a alumnos y profesores para saber cuáles son las percepciones sobre el tema y las medidas aplicadas. Así se recogen una serie de medidas adoptadas por los profesionales entrevistados tales como la utilización de una letra especial o fragmentar los textos para leer.
\end{abstract}

Palabras clave: dislexia, lengua inglesa, metodología, intervención, adaptación, condiciones de aprendizaje, proceso de aprendizaje, dificultad de aprendizaje.
Abstract: Dyslexia is a Learning Disorder with a great impact in every area of the official curriculum, especially those involving the development of communicative competences. This situation is particularly acute in relation to the first foreign language area: English. This language is increasingly widespread in our classrooms as a result of the establishment of bilingual schools. Despite the general methodological adaptation applied within the subject of English as a foreign language, when there are students with special educational needs in the classroom no specific adaptations are usually carried out. Furthermore, the existing guidelines and protocols provided by educational inspectors are rarely, if ever, applied. The intention of the current research is to compile the range of methodological measures established by educational inspectors, as well as other professionals, with the aim of providing the best education to students with special educational needs in an inclusive way. For the purposes of this research, we analysed three case-studies involving three different students with learning disabilities. We conducted interviews not only with parents, but also with other students and teachers, in order to better understand what are the perceptions about the topic and the effectiveness of the learning adaptations applied. We recommend the adoption of some of the measures proposed by the interviewed professionals. Among these, using special lettering or breaking down the texts to be read into smaller sentences are proving ones of the most effective.

Keywords: dyslexia, English language, methodology, intervention, adaptation, learning conditions, learning process, learning difficulty. 
La educación es un campo que debe ir acorde a las cambiantes características de la sociedad para poder dar respuesta a las necesidades de esta. Así nos encontramos ante una diversidad social con tendencia a la complejidad que necesariamente exige una respuesta acorde a sus necesidades (Pérez, 2017). La lectura y la escritura son herramientas que sirven para vehicular la información (Pannetier, 2017) y en la sociedad actual la lectura es una capacidad fundamental para el día a día. "Ya sea el modo de empleo de un electrodoméstico que acabamos de comprar, las consignas de un cajero automático o el contrato de un seguro, la lectura forma parte de la vida cotidiana" (Pannetier, 2017, p.9). De la misma manera la capacidad de escritura es fundamental para realizar las gestiones básicas en el desarrollo diario e incluso para mantener un negocio. Por lo tanto, son dos capacidades imprescindibles para el desarrollo pleno de la persona en la sociedad. Tener un buen nivel de competencia lectoescritora será el pilar de todo el desarrollo posterior del niño y la niña (Sánchez, 2010).

La dislexia se incluye dentro de los trastornos del neurodesarrollo como un trastorno específico del aprendizaje con dificultad en la lectura y/o en la expresión escrita (American Psychiatric Association, 2014). Este trastorno se encuentra entre las dificultades de aprendizaje más comunes y tienen graves consecuencias escolares (Judica, De Luca, Angelelli, Spinelli y Zoccolotti, 2017). Aunque se pueden encontrar diversas definiciones para la dislexia vamos a exponer las principales al ser las más aceptadas por la comunidad científica. De acuerdo con la asociación internacional de la dislexia (IDA por sus siglas en inglés),

la dislexia es una dificultad específica de aprendizaje de origen neurobiológico. Se caracteriza por dificultades con el reconocimiento preciso y / o fluido de palabras y por la deficiente ortografía y descodificación. Estas dificultades típicamente resultan de un déficit en el componente fonológico del lenguaje que a menudo es inesperado en relación con otras habilidades cognitivas y la provisión de instrucción efectiva en el aula. Las consecuencias secundarias pueden incluir problemas en la comprensión lectora y una experiencia de lectura reducida que pueden impedir el crecimiento del vocabulario y el conocimiento previo (International Dyslexia Association, 2002, definición).

De acuerdo con la Federación Mundial de Neurología (WFN por sus siglas en inglés) la dislexia es "un trastorno en niños que, a pesar de (recibir) instrucción educativa convencional no logran las habilidades lingüísticas de lectura, escritura y ortografía esperadas, en consonancia con sus habilidades mentales." (Change Dyslexia, 2016). La causa de la dislexia del desarrollo aún se desconoce a pesar de décadas de intensa investigación (Huettig, Lachmann, Reis y Petersson, 2017).

\section{Dislexia y aprendizaje de una segunda lengua}

Paralelamente a la importancia otorgada a la lectoescritura, la sociedad actual premia a las personas con un dominio medio-alto de algún idioma extranjero, especialmente en cuanto al inglés se refiere ya que se ha posicionado como uno de los idiomas más importantes a lo largo de todo el mundo (Verplaetse y Migliacci, 2017) puesto que está presente en prácticamente todas las relaciones comerciales que tienen lugar hoy en día. Igualmente, la adquisición de una segunda lengua es una ciencia joven, en la medida en que lucha por emerger y diferenciarse de campos como la educación y la lingüística aplicada (Long, 2017). Es por ello por lo que la educación bilingüe ha sido una opción educativa en muchos países durante más de 50 años, aunque sigue siendo controvertida, especialmente en términos de su idoneidad para todos los niños (Bialystok, 2016).

\section{Dislexia y educación bilingüe en España}

En España desde hace más de una década las administraciones educativas han impulsado la implantación de programas de educación bilingüe y, por tanto, estos han proliferado en los últimos años en las etapas de educación infantil, primaria y secundaria (García, 2015; Jover, Fleta y González, 2016; Pavón, 2018). En el año 2013 se estimaba que participaban en algún programa bilingüe en este país en 
torno a un millón de alumnos en más de 5.000 colegios públicos de educación primaria y 2.000 institutos de educación secundaria, y que la tendencia predecible era que este número iría en aumento (British Council, 2015). Tomando como referencia la Comunidad de Madrid, el objetivo para el año 2015 era contar con más de 3000 profesores habilitados y el 100\% de los centros de educación infantil, el $50 \%$ de los centros de educación primaria y el $30 \%$ de los centros de secundaria y bachillerato completamente desarrollados como centros bilingües (Gerena y Ramírez-Verdugo 2014). Contextualizando en la etapa de primaria, según los datos del Ministerio de Educación, Ciencia y Deporte para el curso 2015-2016 las comunidades autónomas con un mayor porcentaje de alumnos que participan en experiencias de aprendizaje integrado son Asturias (50,8\%), Castilla y León (48,2\%), Castilla La Mancha (41,1\%) y Madrid (40,2\%).

En España la mayor parte de los programas de educación bilingüe se basan en el que se ha venido a denominar aprendizaje integrado de contenidos y lengua (AICLE en español) conocido por sus siglas en inglés como CLIL (Content and Language Integrated Learning). En este tipo de enfoque, los contenidos académicos se enseñan a través de una lengua extranjera con el fin de que el aprendizaje de esos contenidos y el de la propia lengua extranjera se desarrolle de forma paralela (Jover et al., 2016; Pavón, 2018). Pero este nuevo enfoque pedagógico no soluciona los problemas de inclusión que se venían arrastrando desde hace años en las escuelas, y en consecuencia en la sociedad en general (Cummins, 2017). Esta situación se produce en el seno de la educación inclusiva, entendiendo que las escuelas inclusivas, han de ser capaces de responder a las necesidades educativas de sus alumnos, ofreciendo a todos las mismas oportunidades educativas y ayudas (curriculares, personales, materiales y de apoyo), necesarias para su progreso académico y personal (Pérez-Jorge, Alegre, Rodríguez-Jiménez, Márquez-Domínguez y de la Rosa, 2016). En este sentido, desde el ámbito educativo es necesario aplicar medidas que ayuden al alumnado con necesidades a mejorar en sus dificultades y que les predispongan a la adquisición de las competencias curriculares a tenor de sus necesidades (Romero, Castaño y Córdoba, 2016). Considerando además que los docentes son una pieza fundamental para garantizar el éxito de la implementación de la educación inclusiva (Flores y Villardón, 2015; Solís, Pedrosa y Fernández-Mateos, 2019). Por lo tanto para que todos los alumnos tengan pleno acceso a la educación de la mejor forma posible independientemente de sus posibles problemas o trastornos es importante no solo conocer las propiedades de la lengua extranjera, sino también dar respuesta a los alumnos con necesidades educativas específicas de una manera integradora e inclusiva dentro del aula.

Los maestros y profesores desconocen casi completamente las diferencias existentes en el tratamiento de determinados trastornos en cuanto a las dos lenguas que nos ocupan se refiere, sin poder, por lo tanto, establecer un entorno inclusivo de aprendizaje para sus alumnos, ni adaptar sus clases y actuaciones a las necesidades reales de los estudiantes (Barrios y García, 2009). Los programas bilingües nos brindan más oportunidades pedagógicas que los programas monolingües, pero solo si nos enfocamos en implementar pedagogías de comunicación (Cummins, 2017). El punto de partida ha de ser un diagnóstico certero de la competencia inicial del alumnado de modo que los objetivos del programa se ajusten a los mismos (Pavón, 2018). Asimismo, es fundamental que antes de iniciar una educación bilingüe nos planteemos el beneficio real para el alumno desde el punto de vista cognitivo (Urraca, 2012).

Si bien la inclusión y su aplicación en los centros educativos es un campo novedoso, su aplicación en el área de inglés lo es aún más. Por ello la bibliografía existente en este ámbito es escasa y poco precisa para el tema que nos ocupa: qué estrategias favorecen los procesos de enseñanza-aprendizaje de los alumnos con dislexia diagnosticada que estudian en centros de Educación Primaria. No obstante, encontramos en el contexto internacional estudios pioneros que señalan una gran variación en la gravedad de los problemas del idioma extranjero y en las diversas habilidades del lenguaje verbal y escrito afectadas en el idioma extranjero (Schneider y Crombie, 2003) y por tanto, la importancia de que las diferencias de estos alumnos deben ser reconocidas y atendidas dentro del sistema educativo (Peer y Reid, 2000). En el contexto educativo español nos encontramos con una ausencia de estudios empíricos que relacionen el rendimiento alcanzado por los alumnos al finalizar el curso escolar y la presencia o ausencia de alumnos con necesidades específicas de apoyo educativo (ACNEAE) en las aulas ordinarias (Arigita, 
2016). Algunos estudios sugieren que el aprendizaje de este alumnado podría verse afectado por una mayor lentitud (Domingo, 2015). Asimismo, nuestro sistema educativo cuenta con mecanismos y herramientas para responder a la educación inclusiva, sin embargo, estos no están desarrollados de forma adecuada y por este motivo no son efectivos ni resuelven los problemas (Medina, 2016).

En primer lugar, se considera apropiado señalar las estrategias utilizadas para trabajar con los alumnos diagnosticados con dislexia recogidas por el Ministerio de Educación y Ciencia en su libro La atención al alumnado con dislexia en el sistema educativo en el contexto de las necesidades específicas de apoyo educativo (2012) entre las que destacan la utilización de recursos diversificados dentro del aula y grupos de refuerzo también dentro del aula por ser las más inclusivas. Otros autores proponen la intervención multisensorial para el acercamiento del currículum a este tipo de alumnado. Aunque los autores no se centran exclusivamente en el área de inglés, esta estrategia puede ser fácilmente aplicable a dicha área. Incluso esto se practica de manera innata en las primeras etapas del aprendizaje de la lengua puesto que es muy habitual el empleo de numerosas estrategias visuales como flashcards y vídeos. Se ha demostrado que el método Orton-Guillingham es muy eficaz para el entreno de la lectura y la escritura en los alumnos con dislexia en su lengua materna (Gómez, 2017). Queda por probar si esta metodología sirviese para el aprendizaje del inglés como lengua extranjera no solo para los alumnos con dislexia, sino que esta sea extensible para el resto del alumnado de ese aula con el fin de lograr un entorno inclusivo.

Otros autores como Labrador (2015) hacen especial énfasis en la repetición de las palabras, en trabajar la sonoridad de estas y en trabajar repetitivamente en la comprensión de las normas ortográficas del inglés. Este proceso puede resultar muy largo y tal y como muestra el propio autor difícilmente realizable con un currículum tan amplio. Por su parte, Di Francesco (2017) ha demostrado en la utilización de hojas de diferentes colores un enorme beneficio para estos alumnos, los cuales retenían mejor la grafía de las palabras en hojas con fondo amarillo en contraste con otros colores.

Finalmente hay que destacar el estudio de Rivas (2016) el cual pone de manifiesto las enormes ventajas de la utilización de las Tecnologías de la Información y la Comunicación para ayudar a alumnos con dislexia.

Como se ha mencionado anteriormente pocas son las propuestas en las que se señale directamente una relación entre un entorno inclusivo y el aprendizaje de una lengua extranjera como el inglés; teniendo que ceñirnos a las estrategias que se han planteado para el aprendizaje del inglés como lengua extranjera. El presente estudio pretende arrojar luz sobre este tema y por ello se plasman las actuaciones reales que llevan a cabo maestros de Educación Primaria especialistas en la lengua extranjera: inglés cuando tienen en sus aulas alumnos con un trastorno específico del aprendizaje de tipo con dificultades en la lectura (dislexia) de acuerdo con el Manuel Diagnóstico de Enfermedades Mentales en su versión más reciente (DSM-V, 2014), cuya prevalencia refleja datos controvertidos, que varían desde el 3.1-3.2\% al $17.5 \%$ (Cuadro, Von Hagen y Costa, 2017).

La presente investigación se ha puesto en marcha tras la notificación de la existencia de un problema conceptual y práctico. Esta investigación pretende profundizar en las estrategias inclusivas que un maestro de inglés en educación primaria practica con un alumno con dislexia y como estas pueden ser extensibles al resto de maestros, entendiendo que cada uno aportará su libertad de cátedra para ejercerlas. Esta investigación se plantea como una primera exploración e investigación previa para una futura investigación más profunda y extensa. Es por este motivo por el que únicamente nos centraremos en un estudio de caso de tres alumnos de $5^{\circ}$ de primaria de un mismo centro y nivel educativo de titularidad pública de la Comunidad de Madrid.

\section{Metodología}

Se ha elegido el estudio de caso para conocer en profundidad la situación y las opiniones de todos los agentes implicados, alumnos, padres y maestros, con el fin de hacer una primera aproximación a la cuestión. Además, se pretende a su vez conocer, comprender y poner de manifiesto diferentes necesidades educativas relacionadas con la materia que nos ocupa. 
Para llevar a cabo la presente investigación se ha contado con la participación de una serie reducida de personas, todas relacionadas en mayor o menor medida entre sí. Se ha contado con tres alumnos diagnosticados con trastorno específico de aprendizaje de tipo dislexia cursando $5^{\circ}$ de primaria, sus padres, especialmente involucradas han estado las madres de los tres; un maestro que les dio clase de inglés y asignaturas bilingües del curso escolar anterior y dos maestros que han impartido clase de inglés como lengua extranjera el presente curso escolar durante el desarrollo de las observaciones. Con todos ellos se ha tenido la oportunidad de realizar las entrevistas y observaciones requeridas por la investigación.

Además, se ha podido contar con las observaciones de la directora pedagógica del centro, la jefa de estudios y la orientadora las cuales aunque no han estado presentes durante el desarrollo de la investigación, sí han realizado aportaciones a los maestros para llevar a cabo estrategias de ayuda a estos alumnos, especialmente en lo que a la legislación compete.

Primeramente, antes de acudir al centro escolar a realizar las observaciones pertinentes, el investigador estableció una serie de cuestiones a las que pretendía dar respuesta:

> ¿Qué tipo de alumnos con necesidades específicas de apoyo educativo tiene mayores problemas en el área de primera lengua extranjera?

> ¿En qué destreza o subdestreza presentan más dificultades los ACNEAE?

, ¿Qué tipo de adaptaciones necesitan estos alumnos en dicha área atendiendo a las cuatro destrezas básicas, especialmente en lo relativo al escuchar y hablar ya que es lo que mayor importancia tiene en el currículum de Educación Primaria?

> ¿Cuál es la mejor manera de ayudar a los alumnos de ACNEAE para alcanzar los objetivos propuestos en el currículum de Educación Primaria en lo relativo al área de primera lengua extranjera?

La recogida de información se ha realizado en diferentes fases atendiendo a la temporalización y evolución tanto del curso escolar como de la programación de aula de los maestros implicados. En términos generales las observaciones en el desempeño de los alumnos se han llevado a cabo durante el primer trimestre del presente curso escolar.

Para la recogida de todos estos datos se han registrado por escrito todas las entrevistas que se han mantenido durante todas las fases, así como se han registrado todas las observaciones llevadas a cabo. A través de los instrumentos de recogida de información se ha hecho un análisis exhaustivo y controlado de los datos lo que ha facilitado su análisis de manera lo más impersonal posible con el fin de no interferir en los resultados. Mediante una técnica descriptiva se ha elaborado un resumen de los datos y de la información recogida para después organizarla. Con el objetivo de preservar el anonimato de los participantes se ha evitado el uso de sus nombres cambiándolos por las iniciales de sus nombres a lo largo de toda la recogida de los datos.

\section{Resultados}

Los datos recogidos en la presente investigación ponen de manifiesto los siguientes resultados:

De los datos recogidos por los padres, estos expresaban que existe un desconocimiento generalizado sobre el trastorno que afecta a sus hijos, creando rechazo ante la problemática especialmente durante el curso escolar en el que se diagnostica a sus hijos. Además, los padres ponen de manifiesto la falta de información para ayudar a sus hijos en casa y apoyar los conocimientos aprendidos en el colegio. En resumen, expresan la falta de conocimientos sobre el trastorno que afecta a sus hijos y por lo tanto la ausencia de una guía que les ayude a comprender y a ayudarlos.

Por otro lado, en cuanto a las estrategias para ayudar educativamente a estos alumnos propuestas por el gobierno, los maestros muestran cierta complejidad para aplicarlas por la falta de formación y de recursos, especialmente temporales, para su correcta ejecución. Además, muestran que estas medidas, aunque ayudan se quedan escasas para tratar con estos alumnos. Asimismo, se muestra 
cierta incongruencia entre algunas de estas medidas y la metodología que desde el mismo organismo se propone para el proceso de enseñanza-aprendizaje de la primera lengua extranjera, puesto que no es viable eliminar todos los elementos distractores del aula si a la vez se pretende que los alumnos estén expuestos a la máxima cantidad de idioma escrito posible.

Además, los mismos maestros exponen su preocupación ante la pérdida de atención de estos alumnos aumentado por la dificultad añadida de seguir las clases si estas se imparten en la lengua extranjera. Por otro lado, los maestros consideran que la ayuda que los profesores de apoyo brindan no solo a estos alumnos sino a todos en general es muy favorable puesto que se trabaja en parejas los contenidos expuestos en las clases, asemejándose a una clase particular.

Una de las grandes aportaciones que la presente investigación ha recogido han sido las estrategias llevadas a cabo por estos maestros que han ayudado enormemente a sus alumnos con dislexia. A continuación, se listan las más representativas:

, Para los ejercicios de lectura se emplea la letra con la que los alumnos se sientan más cómodos (Arial, por ejemplo) en un tamaño algo mayor a la habitual.

, Además, en este tipo de ejercicios de lecturas de extractos de textos, estos se le proporcionan fragmentados a los alumnos; haciendo coincidir el fragmento de texto con la pregunta que tienen.

, A sabiendas de los problemas que estos alumnos tienen en su lengua materna con el deletreo de las palabras, se pasan por alto pequeños fallos de ortografía de las palabras siempre que estas coincidan en su sonoridad con lo que el alumno ha escrito.

, En términos generales, los tres alumnos destacan por sus grandes habilidades en las destrezas de escuchar y hablar, por lo que se potencian estas dos para evaluar la comprensión de vocabulario y de los aspectos gramaticales.

- A la hora de realizar ejercicios de escucha donde los alumnos tienen que leer las preguntas, o bien los profesores de apoyo o bien el propio maestro lee los enunciados de estas cuestiones oralmente puesto que la lectura no es lo que se está trabajando en ese momento. Se ha notado enorme mejoría en este tipo de ejercicios utilizando esta estrategia. Además, se procura cambiar las preguntas escritas de estos ejercicios por imágenes, mucho más sencillas de reconocer por este tipo de alumnado.

, El maestro provee a los estudiantes de determinadas herramientas facilitadoras de su aprendizaje entre las que destacamos proporcionarles resúmenes claros y precisos de los elementos teóricos que se tienen que estudiar (aunque estos vienen en el libro de manera menos concisa); así como hojas de vocabulario en las que se establezca una asociación clara entre la palabra escrita y un dibujo.

, Durante los exámenes o extensos ejercicios de lectura comprensiva se les permite a estos alumnos tapar el resto de las preguntas que no estén contestando en ese momento con una hoja de papel en blanco, con el objetivo de centrar su atención sobre la pregunta concreta que están llevando a cabo en ese momento.

, El uso de diferentes colores para asociar los tipos de palabras (sustantivos, adjetivos, etc,) o para asociarlos con otros ítems también ha demostrado ser de gran utilidad.

, El empleo de técnicas de gamificación permite a estos niños mantener su motivación alta por el trabajo llevado a cabo en el aula.

, En cuanto al empleo de palabras pegadas en las paredes de la clase, se ha procurado encontrar un punto medio entre la propuesta de eliminarlas y la metodología de dejarlas; manteniéndolas en el aula, pero solo en la pared final del aula en donde el alumnado no las ve durante el desarrollo de las clases, pero sí les presta atención durante los intercambios de clases u otros momentos de ocio. 
, Finalmente, aunque se ha demostrado que el empleo de las nuevas tecnologías de la información y la comunicación ayudan enormemente a estos alumnos, debido al desconocimiento de aplicaciones concretas de los maestros, de la falta de recursos tanto materiales como temporales y de la gran cantidad de contenidos a cubrir en un solo curso escolar no se han llevado a cabo proyectos destacables con el uso de estas tecnologías.

\section{Conclusiones}

En cuanto a los resultados cuantitativos de los tres alumnos estudiados en esta área cabe decir que han sido satisfactorios, contando con la ventaja de que en la lengua extranjera es de las pocas asignaturas en las que el examen cuenta con una parte oral. Estos alumnos sacan gran provecho de esta parte oral de los exámenes compensando la nota de la parte escrita. Estos resultados están en la línea de los hallados por Labrador (2016) y su énfasis en la repetición de las palabras y la sonoridad. Por tanto, en alumnos con dislexia podemos decir que el bilingüismo no ocasiona más problemas, sino que su aprendizaje podría verse ralentizado al estudiar varias lenguas al mismo tiempo ya que supone unas dificultades añadidas (De Marco, 2010; Domingo, 2015).

Se ha demostrado que las dos destrezas que presentan menos dificultades para estos alumnos son la escucha y el habla, aunque no se ha podido comprobar cuáles son las mayores dificultades de estos alumnos con cada una de las cuatro destrezas al necesitar este objetivo una investigación propia más en profundidad. Asimismo, se han descubierto numerosas estrategias que han demostrado ser eficaces con estos alumnos y que facilitan el acceso al currículum a estos alumnos, recordemos las ventajas del uso de las Tecnologías de la Información (Rivas, 2016).

Se ha descubierto la poca formación que tienen los maestros de Educación Primaria sobre la atención de este tipo de dificultades de aprendizaje y cómo los organismos oficiales son de escasa ayuda para trabajar con estos alumnos. Este hecho coincide con otros estudios que señalan que la falta de formación del profesorado es un impedimento para aplicar con éxito el modelo inclusivo (Avramidis y Kalyva, 2007; Cagran y Schmidt, 2011; Lee, Tracey, Barker, Fan y Yeung, 2014).

Finalmente cabe destacar los datos arrojados por los propios estudiantes cuando afirman que, aunque su motivación es alta para el estudio de esta asignatura pues ellos son plenamente conscientes de que su mayor ventaja es la presencia de la citada evaluación oral, se sienten muy confusos con los libros de texto de esta área puesto que suelen ser muy coloridos con multitud de imágenes desordenadas. Sin embargo, conviene recordar que Di Francesco (2017) apunta al beneficio de la utilización de hojas de diferentes colores.

A la hora de diseñar y llevar a cabo la presente investigación se han detectado diversas limitaciones entre las que destacamos las dos siguientes. Los datos de la muestra se corresponden a estrategias de maestros que ejercen la docencia en España exclusivamente, en centros presenciales con limitado acceso a las nuevas tecnologías individualizadas para los alumnos. Por lo tanto, se considera que los datos de la presente investigación tienen fecha de caducidad si se proveen a los centros educativos con los recursos tecnológicos que se exigen para ir en consonancia con los reclamos de la sociedad.

Otra gran desventaja que se ha encontrado con la presente investigación es la imposibilidad de generalizar los hallazgos puesto que se ha centrado en el estudio de tres casos concretos, con unas características personales muy concretas.

A pesar de todo ello, se considera la presente investigación como el punto de partida para profundizar más detenidamente en todos los aspectos aquí expuestos, evaluándolos individualmente y en su combinación. Se considera esta investigación como una primera aproximación a las estrategias y pensamientos de los diferentes agentes implicados en los procesos de enseñanza-aprendizaje de alumnos con diagnóstico de dislexia para seguir trabajando en lograr una plena inclusión de estos alumnos y proponer e implementar nuevas estrategias que favorezcan no solo a los alumnos con dislexia y a sus maestros, sino a todos los alumnos presentes en las aulas de Educación Primaria. 
American Psychiatric Association. (2014). Manual diagnóstico y estadístico de los trastornos mentales $D S M-V$. APA.

Arigita, A. (2016). Estudio de los efectos que tienen los programas madrileños de educación bilingüe sobre el rendimiento académico de los alumnos de primaria con necesidades específicas de apoyo educativo. Verbeia, 1, 39-56. https://bit.ly/36nV3ss

Avramidis, E., y Kalyva, E. (2007). The influence of teaching experience and professional development on Greek teachers' attitudes towards inclusion. European Journal of Special Needs Education, 22(4), 367-389. https://doi.org/10.1080/08856250701649989

Barrios, E. y García, J. (2009). Las dificultades para la educación integradora desde la perspectiva de futuros maestros de inglés. Relieve, 15 (1), 1-24. https://doi.org/10.7203/relieve.15.1.4185

Bialystok, E. (2016). Bilingual education for young children: review of the effects and consequences. International Journal of Bilingual Education and Bilingualism, 1-14. https://doi.org/10.1080/13670050 .2016 .1203859

British Council (2015). Formación inicial para profesores de programas bilingües en inglés: políticas, prácticas y recomendaciones. British Council.

Cagran, B. y Schmidt, M. (2011). Attitudes on Slovene teachers towards the inclusion of pupils with diferrent types of special needs in primary school. Educational Studies, 37 (2), 171-195. https://doi. org/10.1080/03055698.2010.506319

Cuadro, A., von Hagen, A. y Costa Ball, D. (2017). Procedural differences in the calculation of the prevalence of reading difficulties in Spanish-speaking school children/Diferencias procedimentales en el cálculo de la prevalencia del retraso lector en escolares hispanoparlantes. Estudios de Psicología, 38(1), 169-197. https://doi.org/10.1080/02109395.2016.1268388

Cummins, J. (2017). Pedagogies of Powerful Communication in CLIL and Bilingual Education [conferencia plenaria]. Congreso Evidence-Based Strategies to Support Bilingual Education in Spain and the US, Salamanca. https://bit.ly/36VPLFe

De Marco, M. (2010). Programas informáticos para trastornos de lectoescritura, Dislexia y/o TDAH. En P. Arnaiz, Ma.D. Hurtado y F.J. Soto (Coords.) 25 Años de Integración Escolar en España: Tecnología e Inclusión en el ámbito educativo, laboral y comunitario (pp. 1-8). Consejería de Educación, Formación y Empleo. https://bit.ly/2AtXIL4

Di Francesco, E. (2017). La enseñanza del inglés como lengua extranjera en alumnos con dislexia evolutiva [Tesis Doctoral, Universidad de Valencia]. Repositorio. https://bit.ly/2yWOhhj

Domingo, B. (2015). Modelos creativos para el tratamiento de las dificultades de comunicación en los colegios bilingües de la Comunidad de Madrid [Tesis Doctoral, Universidad Nacional de Educación a Distancia España]. Repositorio institucional. UNED. https://bit.ly/2A05wyu

Flores, L. y Villardón, L. (2015). Actitudes hacia la inclusión educativa de futuros maestros de inglés. Revista latinoamericana de educación inclusiva, 9 (1), 63-75. https://bit.ly/302OFpp

García, M. P. (2015). Efectos de la educación bilingüe España, propuestas de mejora y recursos didácticos relacionados con la metodología AICLE [Tesis Máster, Universidad Politécnica de Madrid]. Repositorio institucional. Universidad Politécnica de Madrid. https://bit.ly/2BoGwS1

Gerena, L. y Ramírez-Verdugo, M.D. (2014). Analyzing Bilingual Teaching and Learning in Madrid, Spain: 
A fullbright Scholar Collaborative Research Project. Gist Education and Learning Research Journal, 8, 118-136. https://doi.org/10.26817/16925777.117

Gómez, A. (2017). Intervención Multisensorial para trabajar la dislexia en educación primaria [Trabajo Fin de Máster, Universidad Internacional de La Rioja]. Repositorio institucional. Universidad Internacional de La Rioja. https://bit.ly/3eM7P7s

Huettig, F., Lachmann, T., Reis, A. y Petersson, K. M. (2017). Distinguishing cause from effect-many deficits associated with developmental dyslexia may be a consequence of reduced and suboptimal reading experience. Language, Cognition and Neuroscience, 1-18. https://doi.org/10.1080/2327 3798.2017 .1348528

International Dyslexia Association (12 de noviembre de 2002). Definition of Dyslexia. Recuperado el 27 de diciembre de 2018 de https://dyslexiaida.org/definition-of-dyslexia/

Jover, G., Fleta, M. T. y González, R. (2016). La formación inicial de los maestros de educación primaria en el contexto de la enseñanza bilingüe en lengua extranjera. Bordón. Revista de pedagogía, 68 (2), 121-135. https://doi.org/10.13042/bordon.2016.68208

Judica, A., De Luca, M., Angelelli, P., Spinelli, D. y Zoccolotti, P. (2017). Early reading treatment in children with developmental dyslexia improves both reading and spelling. BPA-Applied Psychology Bulletin (Bollettino di Psicologia Applicata), 65(279).

Labrador, R. (2016). La dislexia en la clase de Inglés como Lengua Extranjera: propuesta de intervención para segundo de bachillerato [Trabajo Fin de Máster,Universidad de Valladolid]. Repositorio institucional. Universidad de Valladolid. https://bit.ly/2XtJzkF

Lee, F.L.M., Tracey, D., Barker, K. Fan, J.C.M. y Yeung, A.S. (2014). What Predicts Teachers' Acceptance of Students with Special Educational Needs in Kindergarten? Australian Journal of Educational \& Developmental Psychology, 14, 60-70. https://bit.ly/2Xonwvv

Long, M. H. (2017). Problems in second language acquisition. Routledge.

Medina, M. (2016). Principales problemas para hacer efectiva la educación inclusiva. Revista de Nacional e Internacional de Educación Inclusiva, 9 (1), 196-206. https://bit.ly/2zUl5lb

Pavón, V. (2018). La controversia de la educación bilingüe en España. Revista Tribuna Norteamericana, 26, 20-27. https://bit.ly/300mgQY

Peer, L. y Reid, G. (2000) Multilingualism, Literacy and Dyslexia: a Challenge for Educators. David Fulton Publishers Ltd.

Pérez, I. (2017). Herramientas para la inclusión: de la educación a la sociedad. Revista Nacional e Internacional de Educación Inclusiva, 10 (2), 13-30. https://bit.ly/2XoBaim

Pérez-Jorge, D. Alegre, O.M., Rodríguez-Jiménez, M.C., Márquez-Domínguez, Y. y de la Rosa, M. (2016). La identificación del conocimiento y actitudes del profesorado hacia inclusión de los alumnos con necesidades educativas especiales. European Scientific Journal, ESJ, 12(7), 64-81. https://doi.org/10.19044/esj.2016.v12n7p64

Rivas, C. (2016). Análisis y evaluación de recursos tecnológicos para la enseñanza y el aprendizaje del alumnado con dislexia [Trabajo Fin de Grado, Universidad de Santiago de Compostela]. Repositorio institucional. Universidad de Santiago de Compostela. https://bit.ly/3dpZDcl

Romero, A., Castaño, C. y Córdoba, M. (2016). Eficacia de un programa de intervención temprana para reducir las señales de riesgo de la dislexia. Revista Nacional e Internacional de Educación inclu- 
siva, 9 (2), 186-200. https://bit.ly/3eNvjZP

Sánchez, M. A. (2010). La iniciación a la lectoescritura en Educación Infantil. Autodidacta: Revista de la Educación en Extremadura, 2, 66-78.

Schneider, E. y Crombie, M (2003) Dyslexia and Foreign Language Learning, David Fulton Publishers Ltd.

Solís, P., Pedrosa, I. y Fernández-Mateos, L.M. (2019). Assessment and interpretation of teachers' attitudes towards students with disabilities. Culture and Education, 31 (3), 576-608, https://doi.org/1 $0.1080 / 11356405.2019 .1630955$

Urraca, A. S. (2012). Trastornos del lenguaje y bilingüismo. En A. Albach (Ed.). Retos de la educación bilingüe (pp.38-75). Ministerio de Educación Cultura y Deporte.

Verplaetse, L. S. y Migliacci, N. (Eds.). (2017). Inclusive pedagogy for English language learners: A handbook of research-informed practices. Routledge.

V.V.A.V. (2012). La atención al alumnado con dislexia en el sistema educativo en el contexto de las necesidades específicas de apoyo educativo. Ministerio de Educación, Cultura y Deporte. 